\title{
Study on Modern Bridge Structure Health Monitoring System Based on Damage Identification
}

\author{
Chunguang HAN, Xu LUO*, Xinping LI, Xinsha FU, Lixiong GU, Zhiyong OUYANG
}

\begin{abstract}
With the rapid growth of traffic, the loads' design of many existing bridges can no longer meet the current vehicle load requirements, and the structural safety is seriously threatened. To ensure the structural safety of the bridge, it is necessary to monitor the bridge health and establish an early warning mechanism to prevent major accidents. The modern concrete bridge structure health monitoring based on damage identification proposed in this paper carried out principal component analysis of modern concrete bridges, and then this paper used principal component analysis (PCA) to locate the nonlinear damage source of the experimental model, which obtained the following conclusions. The maximum shear stress of the steel beam web is about $80 \mathrm{MPa}$, and the bulk stress of steel is reached at $7.5 \mathrm{MPa}$. Furthermore, to reduce the original data's dimensionality, PCA effectively retains the characteristic information of the original data; empirical examples from external factor are presented. The major advantage of applying this framework is that the structural damage identification is simple and reliable with its advantages of dimensionality reduction, noise reduction, and exclusion of out-of-bounds interference factors.
\end{abstract}

Keywords: damage identification; health monitoring; modern concrete bridge; principal component analysis; structural safety

\section{INTRODUCTION}

With the development of the world economy and the needs of the human living environment, various large-scale civil engineering structures have emerged in large numbers. Although these buildings in service have a specified engineering design life, due to sudden conditions (such as earthquakes, rainstorms, typhoons, etc.) or long-term accumulation (such as fatigue, erosion, aging, etc.) they cause different levels of damage to civil engineering structures. If the damage of important components accumulates to a certain level, without early detection and treatment the damage will continue to spread, which will cause the bridge structure to show different levels of damage and deterioration before it reaches the design life, which will affect the operation of the bridge structure [1, 2]. Traditional structural maintenance inspection methods have high costs, high subjectivity, high uncertainty, low efficiency, lack of scientific quantitative basis, which are far from meeting the shortcomings of engineering practice. This paper adopts modern sensing, control, artificial intelligence, communications, materials [3], and data analysis to monitor the structure safety which is more quantifiable, and has become a development trend in the field of modern structure full life health monitoring technology.

Structural damage diagnosis is to detect and evaluate the structure, determine whether there is damage to the structure. It analyses the level and location of the damage, and evaluates the status and trend of the failure. Panesso et al. [4] have carried out the theoretical and experimental studies on the identification of structural damage characteristics, the status of structural work, and the location of structural damage, health monitoring sensor systems, large-scale monitoring data analysis technology, and machine vision measurement. The research results of this kind have been successfully applied to various engineering fields at home and abroad. Xiaodan, F. [5] implemented the remote health monitoring for $3 \mathrm{D}$ visual bridge based on image data analysis, combining the latest achievements in applied mathematics, information technology, and artificial intelligence, which can be achieved unattended on site and save labour. Li et al. [6] studied structural monitoring data, vehicle load spatial distribution, and structural state safety assessment under complex coupling based on compressed sensing theory. Abdeljaber et al. [7] used the long-term health monitoring system big data analysis technology to study the anomaly data mining and the time-varying reliability analysis of the structure under multi-scale correlation. Martinez-Luengo et al. [8] used artificial neural network improved algorithm to predict and quantitatively analyze the structure life cycle health monitoring data. Based on computer vision measurement, Zhiwei Chen et al. [9] used non-contact measurement technology to identify structural mechanical performance parameters. Bas et al. [10] used the fractional total variation theory to deblur the visual measurement image. In summary, the research on existing theories of structural health monitoring mainly uses PCA for damage identification, which identify structural static or dynamic characteristics parameters. The experimental analysis adopts the measured response of the structure to perform damage identification and state assessment under the complex load and environmental coupling. These methods have clear physical meaning. They have been widely used in practical engineering, but there are problems in adaptability, robustness, parallelism, and sensitivity for bridge structures with complex damage sources [11]. In addition, it is difficult to mine anomalous information with massive monitoring data. The response is difficult to reflect the damage and the noise interference during the test in signal transmission. Damage identification factors required to have certain robustness and sensitivity to the error, which can truly reflect the structure attribute. The new system can recognize damage and has high sensitivity for local damage.

Structural geometric deformation monitoring is a key component of bridge engineering health monitoring for the bridge structure status. Abnormal changes in structural geometry can truly reflect the current safety status of the bridge structure to a certain extent. The occurrence of different levels of damage or disease will be truly reflected in the abnormal changes in the bridge geometry. Local damage recognition is one of the damage recognition 
methods. Local damage identification methods include magnetic field method, ultrasonic method, eddy current method, and temperature field method. These methods need to know the approximate location of the damage in advance. For large and complex structures that are not easily accessible by people, it is difficult to use this method to damage detection [12]. Because of these defects, the damage detection has developed. Although the use of static test data is relatively easy to obtain, accurate and stable compared to dynamics, problems such as limited applied load conditions, small measurement information, and difficult or unrealistic loading of actual structures are inevitable [13]. Therefore, the research of damage identification methods has become a hotspot for many scholars at home and abroad. The basic idea is to use the physical properties of the structure.

At present, in the monitoring of long-span bridge structures, structural geometric deformation monitoring mainly forms a sensor array by arranging a limited number of contact sensors at key monitoring points of the bridge structure, which forms a health monitoring system with sensors. Environmental effects are monitored, or total manual, level, and theodolite are used to conduct regular manual inspections at monitoring and control points in the bridge area. Structural health monitoring is not economical, sensors need to calibrate regularly, and monitoring control points are subject to the topographic conditions of the structural area. Based on the above research, this paper proposes a research method of modern concrete bridge structure health monitoring by damage identification. The health monitoring of modern concrete bridge structures truly reflects the true deformation of the structure under load. Compared with the health monitoring system using advanced sensors, the holographic deformation of the bridge structure obtained is more sensitive to local damage, and it overcomes the limitation caused by the number of sensors. The point response data is discrete, which can reflect the global damage and local damage information of the structure.

\section{BRIDGE STRUCTURAL HEALTH ASSESSMENT \\ 2.1 Structural Damage Identification}

The acceleration response $x_{i}(t)$ of the measuring point of the bridge structure $i$ is a random process, which can be expressed as follows:

$$
\begin{aligned}
& x_{i}(t)= \\
& =\varphi_{1} x(t-1)+\varphi_{2} x(t-2)+\varphi_{3} x(t-3)+, \ldots,+\varphi_{p} x\left(t_{p}\right)+u(t)
\end{aligned}
$$

where $\varphi_{j}(j=1,2,3, \ldots, p)$ is the autoregressive parameter, $u_{i}(t)$ is the white noise process, and $u_{i}(t)$ obeys $x_{i}(t)$. Then $\operatorname{NID}\left(0, \sigma^{2}\right)$ is called $p$-order autoregressive process, which is defined as AR model, and is represented by $\operatorname{AR}(p)$. Introducing the lag operator $\Phi(L)$, the above formula can be expressed as follows.

$$
\left(1-\Phi_{1} L_{1}-\Phi_{2} L_{2}-, \ldots,-\Phi_{p} L_{p}\right) x_{i}(t)=\Phi(L) x_{i}(t)=u_{i}(t)(2)
$$

In the formula above, the root mean square error (RMSE) is used to measure the deviation between the observed value and the true value. For a random time series $x$ with n observations, the formula for calculating the root mean square error is as follows.

$$
\operatorname{RMSE}(\hat{x})=\sqrt{\frac{1}{n} \sum_{i=1}^{n}\left(x_{i}-\hat{x}_{i}\right)^{2}}
$$

In the formula, $x_{i}$ represents the exact value and $\hat{x}_{i}$ represents the predicted value. By the AR model established under the state of structural health to predict the response signal when the structure is in an unknown state, due to the time-varying characteristics of the structure, the residual of the AR model will increase, which will cause the root mean square error to increase. For the test sample $y(t)$ in an unknown state, the residual is $u_{t}^{\prime}$, then:

$u_{t}^{\prime}=\Phi_{\mathrm{L}} y(t)$

Combining with Eq. (3), the root mean square error has been obtained in the unknown structure. This paper adopts the dimensionality reduction idea of principal component analysis, where each principal component is a linear combination of the original variables, so that they retain as much information as possible of the original variables, and the principal components are not related to each other. The purpose of principal component analysis is twofold. One is to compress the data, and the other is to interpret the data. By this method, the complex factors can be reduced to several principal components, and redundant information is eliminated, so that complex problems can be simplified, more scientific information can be obtained, the model can better reflect the real situation [14]. The level of interpretation of the original information by a principal component can be measured according to the characteristic value of the principal component. If the characteristic value is larger, it means that the variance contribution rate of the principal component is larger, and the effective interpretation of the original information is stronger. Variance contribution rate can be used as an important criterion for selecting the number of principal components. The basic flow of principal component analysis is shown in Fig. 1.

\begin{tabular}{l} 
Normalize the raw data matrix \\
$\begin{array}{l}\text { Find the correlation coefficient } \\
\text { matrix of standardized data }\end{array}$ \\
\hline
\end{tabular}

Find the eigenvalues and eigenvectors of the correlation coefficient matrix

Determine the number of principal components
according to the cumulative contribution rate of
the characteristic value (greater than $85 \%$ )
Principal Component Matrix
Figure 1 Flow chart of principal component analysis

Technical Gazette 28, 1(2021), 151-159 


\subsection{Data Preprocessing Module}

The sensor data collected during the long-term monitoring of the bridge will inevitably reduce its accuracy due to various factors. Therefore, in order to provide a basis for bridge safety assessment, the quality of the data will be evaluated, that is, the credibility of the data will be given level. The main evaluation methods are as follows.

The acquisition device determines the reliability of the sensor data.The time series analysis method is used to establish the historical trend model of each sensor data, that is, after collecting data for a period, and referring to other bridges' similar data models, a historical trend model is established for each sensor [15]. According to this model to predict the range of data, any data outside this range will be judged as abnormal data. This paper determines the relevance of this data with other data, which establishes relevant models for various types of sensors on each section. When making judgments, suspicious data can be determined based on changes in other data. Based on the data measured by sensors of the same type and close positions, a gray correlation method is used for correlation analysis calculation [16]. If the probability standard deviation of the correlation level obtained by the correlation analysis is less than the threshold value, it indicates that the data measured by different sensors have good correlation, which proves that the sensor is working normally. Otherwise, it means that the data correlation is not good and a certain sensor is working abnormally.

\subsection{Structural Safety Early Warning Module}

Early warning indicators can be set using a model-free method based on statistical data, a method based on a finite element model, or directly set based on experience, standards, and specifications. In bridge monitoring, a single early warning condition (single indicator early warning) is greatly affected by accidental factors and prone to false alarms. Therefore, it is recommended to use a combination of early warning conditions (multiple indicator early warning) to trigger the alarm. Fig. 2 shows an alarm method that triggers an early warning only after three consecutive overruns, which can reduce the false alarm rate to a certain extent.

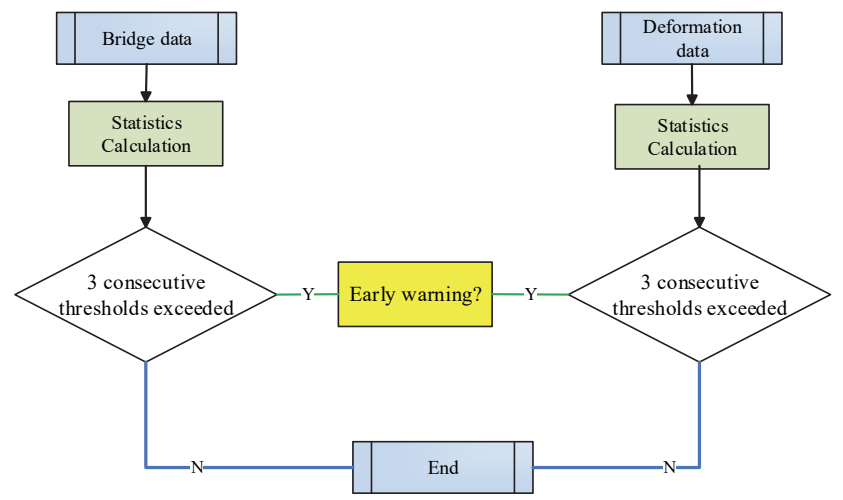

Figure 2 Bridge monitoring and early warning process

The maximum (minimum) value, mean value, variance, standard deviation, and amplitude of change are often used as the input values for primary warning. When monitoring bridges, different statistical sections need to be adopted for different monitoring parameters. For static parameters, the real-time requirements are relatively low, and "day" can be used as a time scale as a statistical section. For dynamic parameters, their high sampling frequency can be "minutes" as a time scale, that is, a statistical value is generated for the data collected every minute, and the statistical value is counted twice.

\section{PROPOSED FRAMEWORK} parts:

The proposed framework comprises the four following

Damage Identification Index adopts PCA (principal component analysis) on the original response data of the structure, which refers to the difference between the original response of the structure and the response of the structure.

Health monitoring of modern concrete bridge introduced the test device and bridge structure, which is used to verify and improve the current geometric monitoring.

Experimental procedure presents four acceleration sensors at the midpoints of each aluminum plate opposite the stimulus source, which are used to measure the acceleration response

The workflow and data analysis for geometric shape monitoring shows the workflow and data processing of the noise reduction and anti-disturbance unit.

\subsection{Damage Identification Index}

After performing the principal component analysis on the original response data of the structure according to the flow chart in Fig. 1, a corresponding load matrix $\boldsymbol{L}$ is obtained. By multiplying the load matrix $\boldsymbol{L}$ and its transposition, a structural overall weight matrix with the same dimensions as the original response data can be obtained. Then the identity matrix is compared with the overall weight matrix of the structure to obtain a comprehensive weight matrix. The meaning of the matrix refers to the difference between the original response of the structure and the response of the structure after transformation by principal component analysis. First, the comprehensive weight matrix $\boldsymbol{G}$ is calculated based on the load matrix.

$\boldsymbol{G}=\left(\boldsymbol{E}-\boldsymbol{L} \times \boldsymbol{L}^{\prime}\right)$

In the Eq. (5), $\boldsymbol{E}$ is the identity matrix, and the error matrix $\boldsymbol{Y}$ of the AR model is multiplied by the comprehensive weight matrix $\boldsymbol{G}$ to obtain the difference between the original response of the structure and the principal component analysis transformation.

$$
\boldsymbol{Y} \times \boldsymbol{G}=\boldsymbol{Y} \times\left(\boldsymbol{E}-\boldsymbol{L} \times \boldsymbol{L}^{\prime}\right)
$$

Then, the data at different sensors in the same state are summed up. In order to eliminate the influence of the positive and negative values, the arithmetic square root is calculated to obtain the index value $D_{1}$. 


$$
D_{1 i}=\sqrt{\sum_{j=1}^{n}(\boldsymbol{Y} \times \boldsymbol{G})^{2}}
$$

where $i$ represents the number of sensors, $i=1,2, \ldots, m . j$ represents the number of tests $j=1,2, \ldots, n$. In order to enhance the sensitivity of the judgment index, the index value $D_{1}$ helps to highlight the difference between the structural damage state and the healthy state, the index value $D_{1}$ is processed as follows.

1) Enhance data sensitivity

In order to highlight the sensitivity of the data, the data in all states of the structure are compared with the data in the reference state to obtain the index value $D_{2}$.

$$
D_{2 i}=\frac{D_{1 i}}{D_{\text {ref }}}
$$

where $i$ is the number of sensors, $i=1,2, \ldots, m$.

2) Finding the critical state of health

Take the index value $D_{2}$ of the structure's health status as the critical value. If the structure sets multiple health conditions, you can arrange the health status index values in ascending order, and then follow a certain guarantee rate $P$ (generally $95 \%$ ), select from small to large.

3) Determine the damage identification index $D$

Subtract the index value $D_{2}$ in all states to be checked from the selected health state critical value to obtain the final damage identification index $D$. If $D$ is greater than zero, the structure is judged to be a damage state [17].

The structural damage recognition process based on the combination of time series and principal component analysis is shown in Fig. 3. The decision principle is bounded by a zero value, a positive value indicates that the structure is in a damaged state, and a negative value indicates that the structure is in a healthy state.

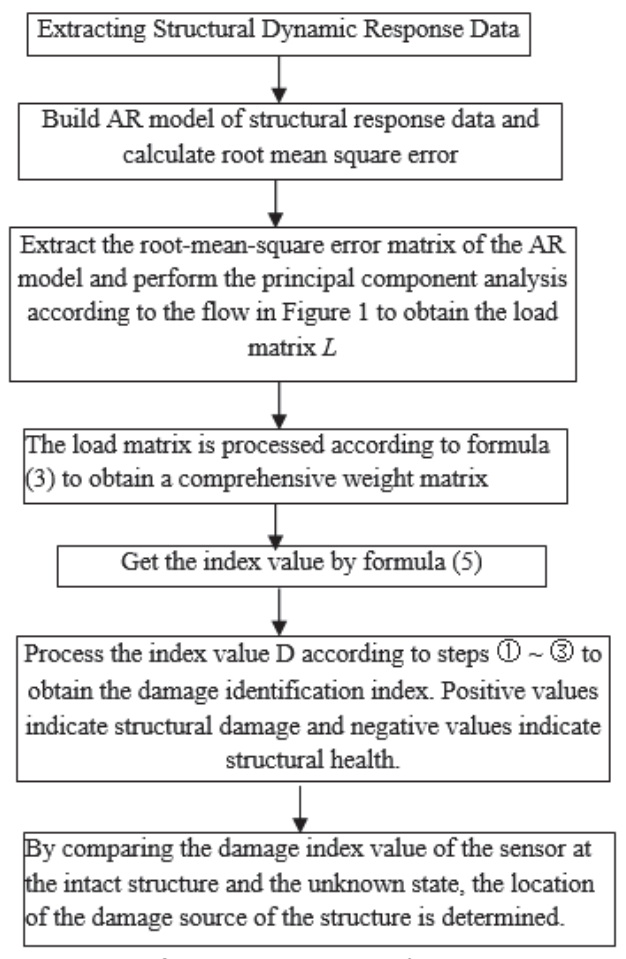

Figure 3 Structural damage identification process

\subsection{Health Monitoring of Modern Concrete Bridge}

Modern concrete bridge structure health monitoring system based on damage recognition is used by modern vision sensor technology, pattern recognition technology, and computer technology. It is mainly composed of active vision sensors, automatic cruise remote control platform, environmental monitoring unit, signal transmission, and communication unit. The structural test device of the standalone automatic bridge monitoring system for modern concrete bridge design and adoption in this paper is shown in Fig. 4.

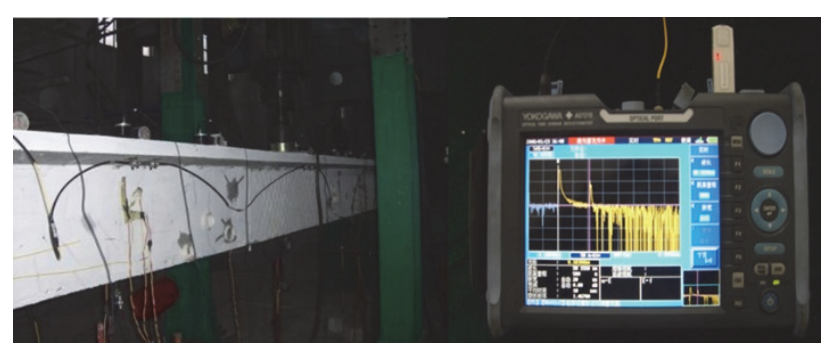

Figure 4 The structure and test device of bridge monitoring system

Based on the existing deep machine vision measurement technology, this paper uses an automatic tour bridge monitoring system test device to test the mechanical behaviour of the modern concrete bridge structure test model in the process of uncertainty load-damage-geometry. The geometrical non-contact remote intelligent sensing method of the test bridge under various damage conditions under uncertain conditions, the test process data structure of the entire process geometric shape monitoring information pool is constructed, and the traditional highprecision contact deformation measuring instrument is used to obtain the test model in each corresponding work. In this case, the measured deformation data of the measurement points is used to verify and improve the current geometric monitoring method.

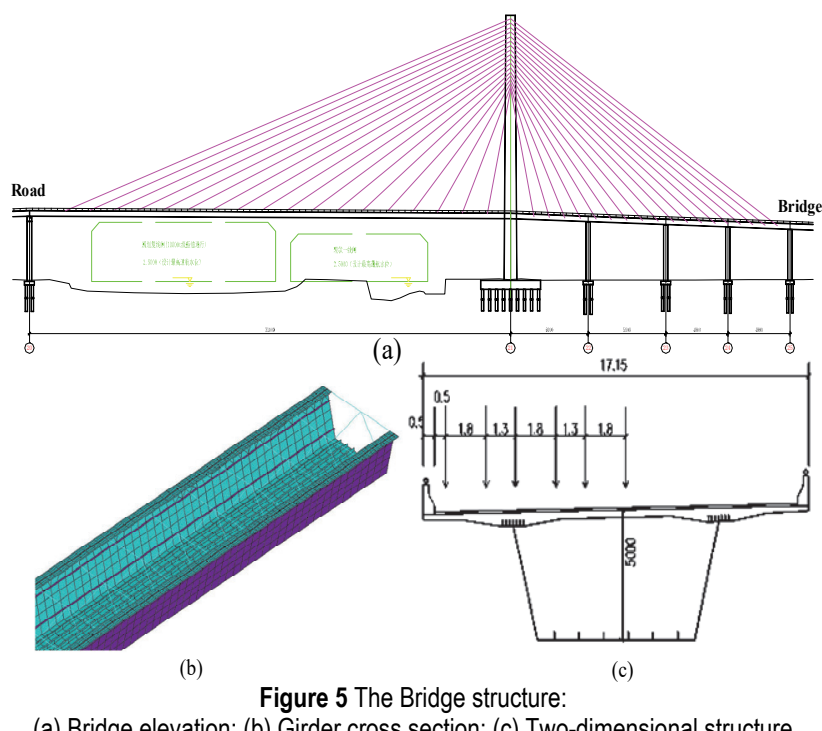

(a) Bridge elevation; (b) Girder cross section; (c) Two-dimensional structure

Fig. 5 shows the structure of bridge health monitoring. Considering that the test model needs to carry out uncertain load loading tests under various damage conditions, structural damage needs to be relatively sensitive to 
changes in geometry. Therefore, the modern concrete bridge deck test, which is easy to repair structural members after damage, is rich in characteristic points of the structure. The bridge monitoring system has studied its structural performance and mechanical behaviour, is the experimental research object.

\subsection{Experimental Procedure}

The modern concrete bridge structural health monitoring is set on the horizontal centerline of the structural floor. The electric exciter provides lateral excitation to the structure floor along the centerline of the structure. A force sensor is connected between the excitation rod and the edge of the structure base plate, which is used to measure the amount of force transmitted by the exciter into the structural system. Four acceleration sensors are set at the midpoints of each aluminum plate opposite the stimulus source, which are used to measure the acceleration response of each layer [18]. The position of the electromagnetic exciter and the linear bearing minimize the torsional vibration of the system. The system is used to collect and process signals, which measure 5 channels of signals from top to bottom. The first channel is the magnitude of the excitation force, and the second channel to fifth channel is the acceleration responses of the structure. The bandwidth of the excitation signal is limited in the range of $20 \sim 150 \mathrm{~Hz}$, which is a kind of stable random excitation signal, which can be approximated as a Gaussian white noise signal. The signal sampling frequency is $320 \mathrm{~Hz}$ and the sampling duration is $25.6 \mathrm{~s}$. 8192 data points were obtained during the sampling process.

When monitoring the geometrical shape of the bridge, the automatic cruise parameters (preset position, watch position, cruise time, sampling time) are set by the computer to remotely control the on-site active vision sensor, environmental monitoring sensor, and obtain the bridge structure monitoring data in the domain. Fig. 6 shows the working process of a modern concrete bridge monitoring system.

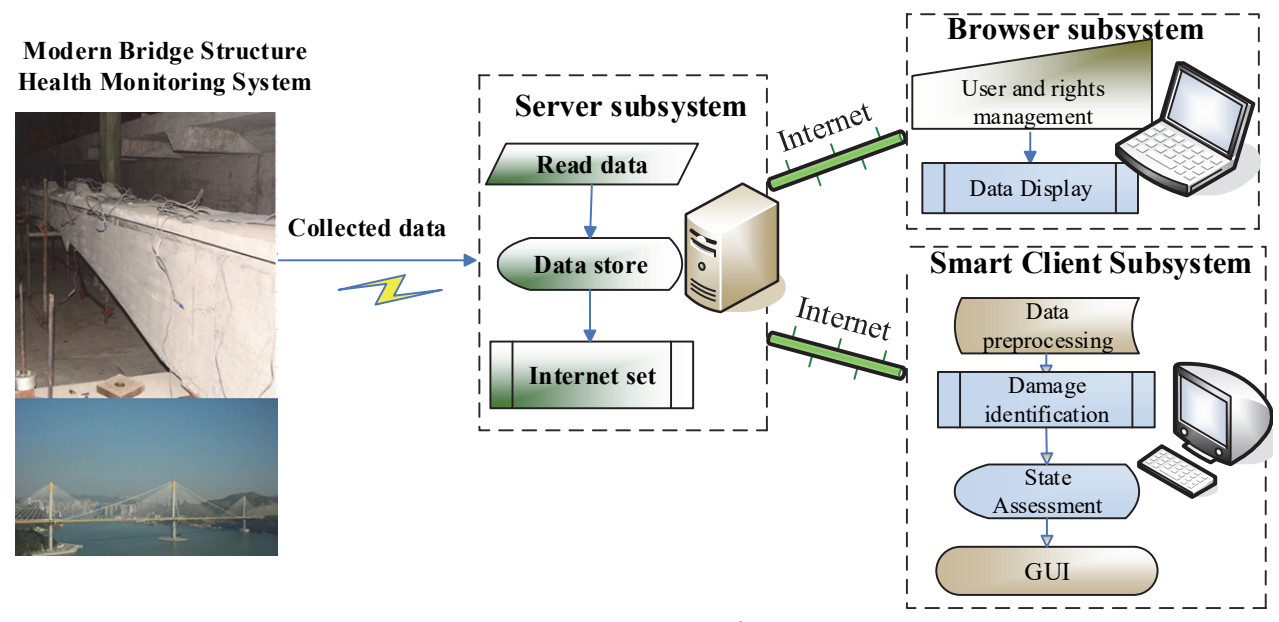

Figure 6 The Experimental procedure of bridge monitoring

\subsection{Workflow and Data Analysis}

The original bridge morphology monitoring data collected by modern concrete bridge structural health monitoring systems based on damage recognition are multi-time-history, multi-angle, multi-view dynamic and static images. The sequence data pool formed by them has strong correlation information in time and space. Therefore, this paper uses hierarchical thinking to build a mathematical model of data sampling and mapping between structural geometric information in turn, performs sampling, noise reduction, anti-disturbance, and Euler motion amplification processing on the original sequence data pool, respectively, to extract the motion information of the structure under random loads.

The sequence data pool composed of the original data has the following characteristics: 1) During the entire experimental process, the morphological data collected in different fields of view have different time history. 2) Multi-view, comprehensive technical and economic analysis, only a small number of devices are used to monitor the local structure in different views, which form local holographic shapes in different views. 3) Multi-angle, automatic cruise remote control platform has different angles when collecting data at each watch position, and equivalent angle conversion is required when processing morphological data. 4) Time and space are strongly related. The time history and spatial information of the original data are affected by the full-bridge random effect of the current moment or period of the structure, and the structural response in the local field of view reflects the overall state of the structure to varying degrees.

Fig. 7 shows the workflow of the noise reduction and anti-disturbance unit. This paper starts from the original signal and establishes a mapping mechanism from the input layer to the output layer. The objective function is used to measure the difference between the output and the true value, and gradually adjust the network structure parameters to make it as suitable as possible for seeking bridge damage parameters.

\section{CASE STUDY}

\subsection{PCA of Bridge Structural Health Monitoring}

The bridge structure health monitoring system has performed 10 experiments under each working condition. Due to the sequence of experiments, external environmental interference during operation, and other 
factors, the acceleration response data obtained each time is different. The AR model created by each experiment result will also be different. In order to reflect the magnitude of this difference, the structural reference stateworking condition was selected, and the principal component analysis of AR models established under the working condition was performed by matlab. Main ingredient means working condition, there are 10 working conditions, and 10 principal components were obtained. The eigenvalue of each principal component in working condition (main ingredient) is shown in Fig. 8.

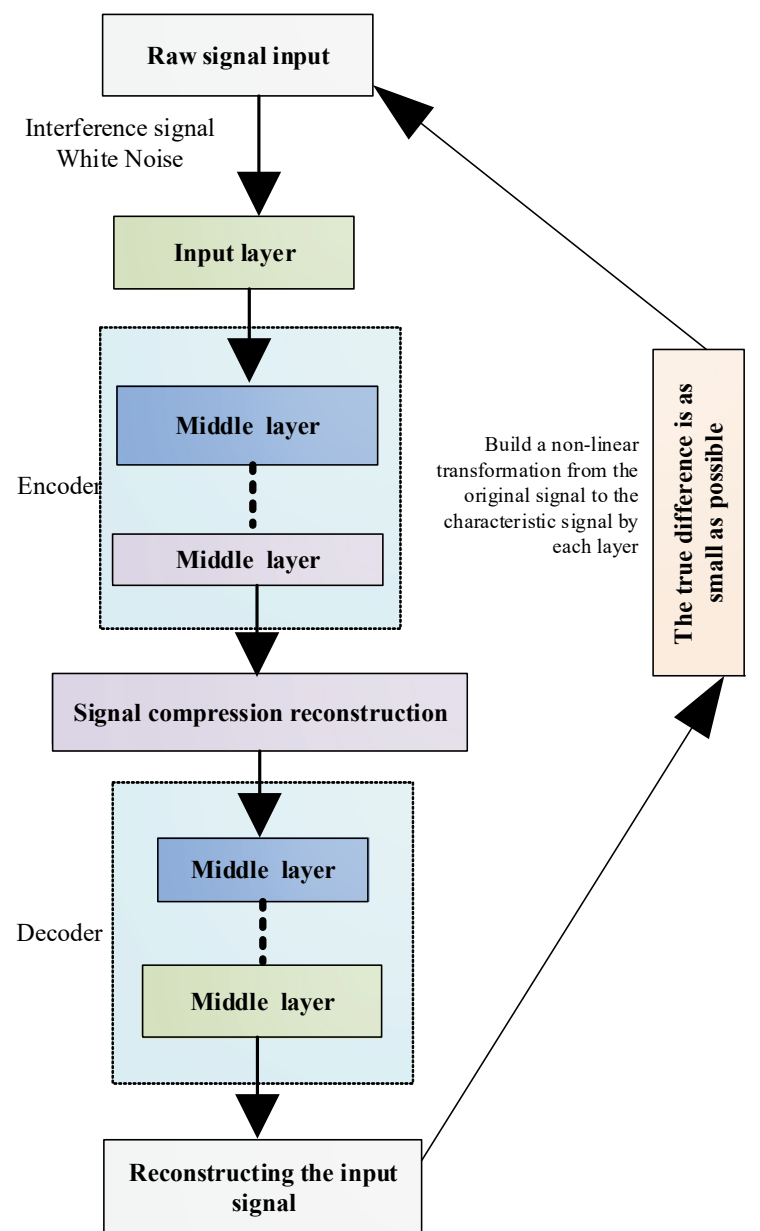

Figure 7 The workflow and data processing of the noise reduction and antidisturbance unit

It can be seen from Fig. 8 that the characteristic value of the first principal component of the working condition 1 is obviously larger than that of the last nine principal components, and the characteristic values of the latter nine principal components are the same. From this, it is obtained that the first principal component in working condition (main ingredient) 1 explains almost all the effective information, that is, the experimental result information, and the last nine principal components explain only a small part of the information. Therefore, it can be concluded that the external environmental interference affected by each experiment is small, and the obtained acceleration response data is very close in size. The experimental conditions are the same, and the differences are only reflected in the sequence of the experimental operations. Principal component analysis was performed on other operating conditions of the experimental model, and the results were very similar to that of operating condition (main ingredient in PCA) 1. Each operating condition can use the first principal component to represent the information of the results of 10 groups of experiments under this operating condition. The original information has been effectively integrated to achieve the dimensionality reduction effect, and the advantages of principal component analysis were reflected.

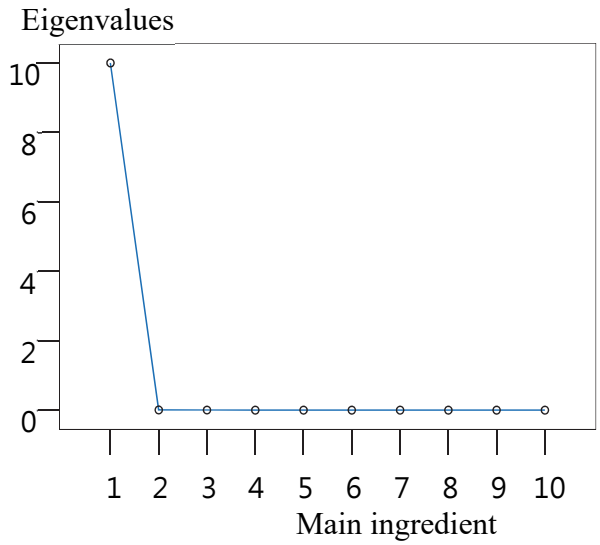

Figure 8 Interpretation of the main component information

\subsection{Normal Forward Stress}

The self-stress of the structure is calculated based on the bulk weight of the material. The bulk stress of steel is $7.5 \mathrm{MPa}$, and the bulk stress of reinforced concrete is -176 $\mathrm{MPa}$. According to the design and construction process, the stress state of the bridge during the construction phase is calculated.

Fig. 10 shows the shear stress envelope of the steel beam web under the action of load combination. It can be seen from the figure that the maximum shear stress of the steel beam web is about $80 \mathrm{MPa}$.

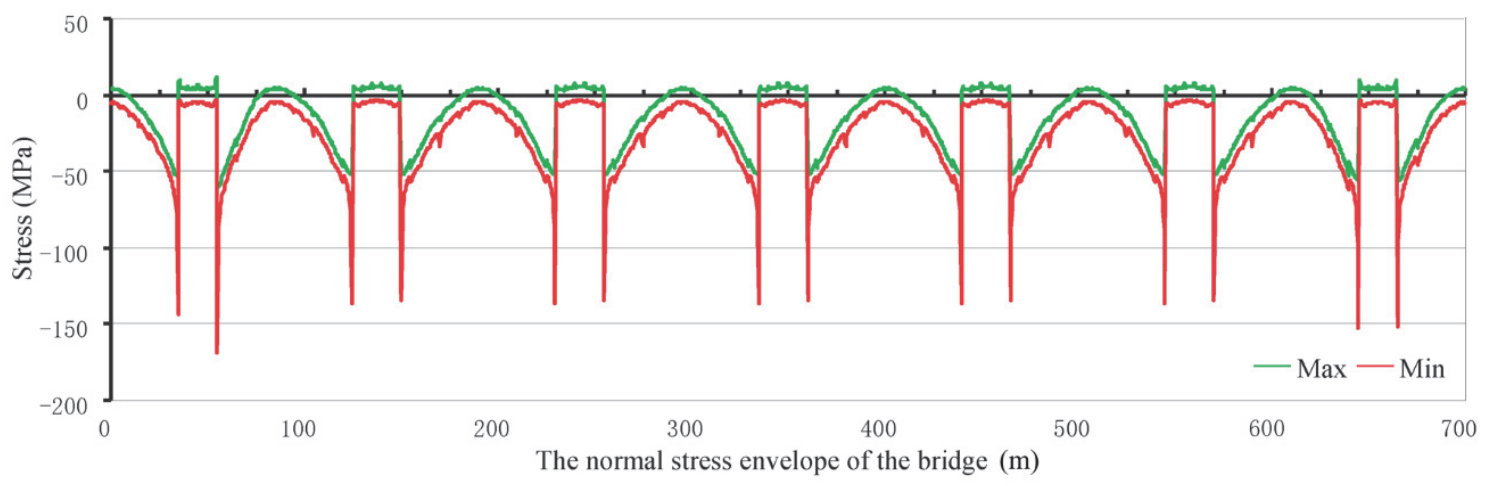

Figure 9 The concrete bridge deck follows the normal stress envelope of the bridge 


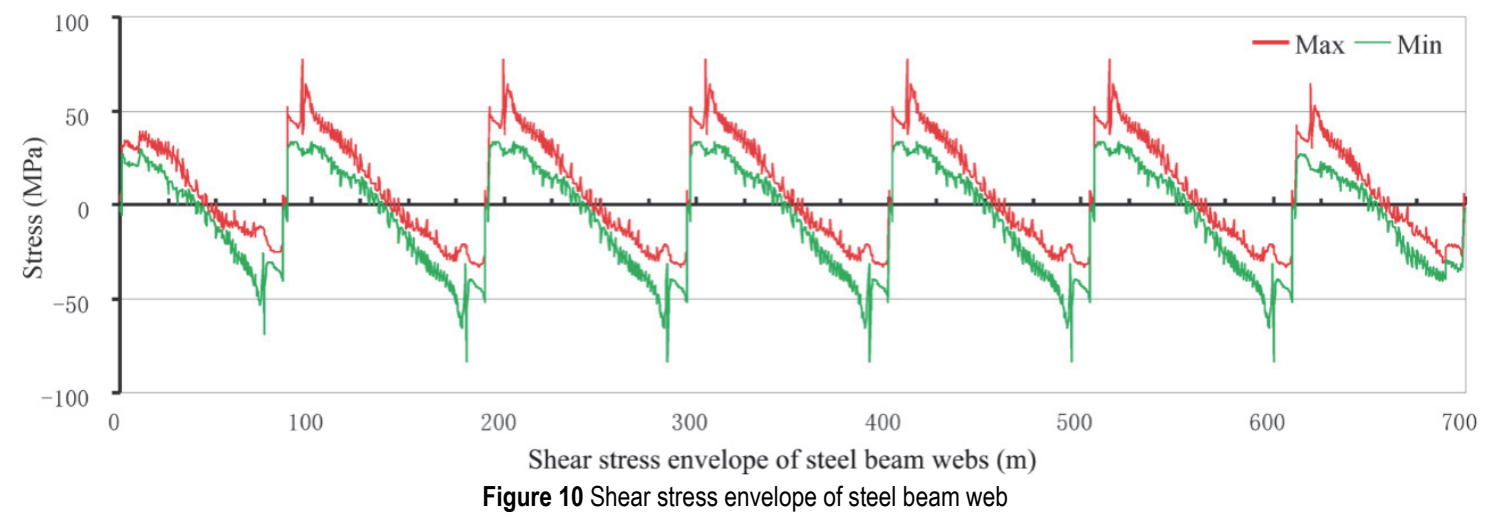

\subsection{Damage Identification}

This paper selects all the acceleration response data of the channel 2 channel 5 of the experimental model to establish the AR model, determine the model order according to the partial autocorrelation function method, and calculate the model residual. According to Eq. (1), the root-mean-square error value matrix of the AR model is obtained, and the matrix is divided into two parts.
One part is composed of the first nine main ingredients (working condition 1 to condition 9) of the rms error matrix without damage. The sample, the other part is composed of the root-mean-square error under all working conditions. The time series and principal component analysis were performed according to the flowchart of Fig. 7, and finally the damage identification index value $D$ of each channel under 10 operating conditions was obtained. The specific results are shown in Fig.11 and Fig. 12.

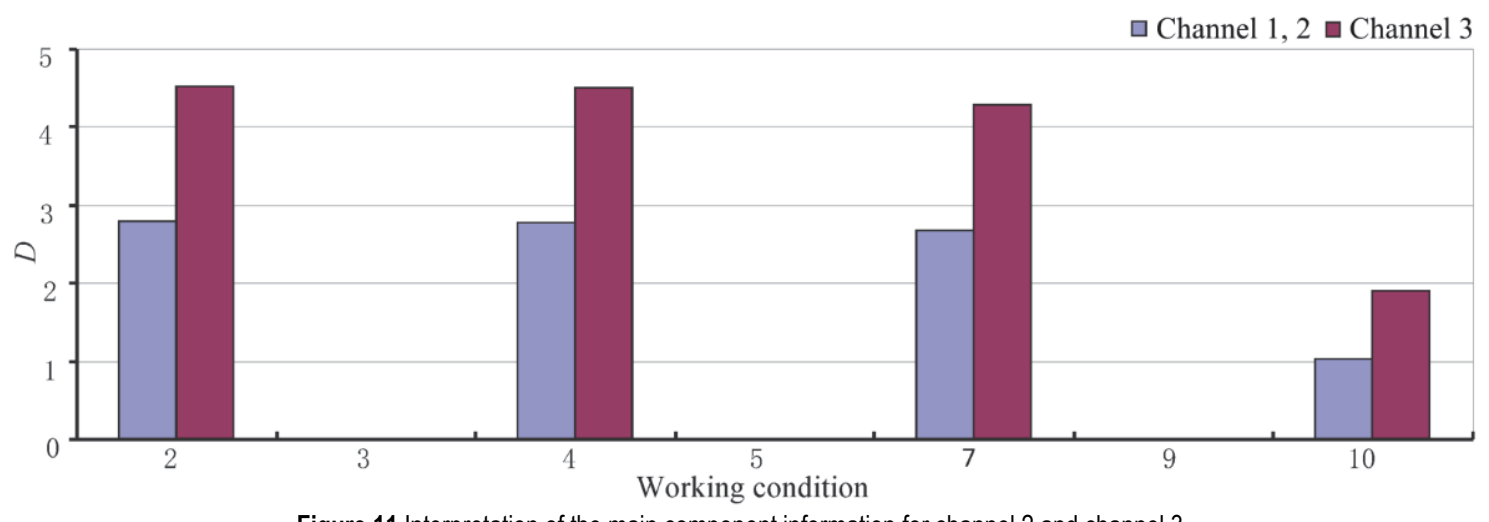

Figure 11 Interpretation of the main component information for channel 2 and channel 3

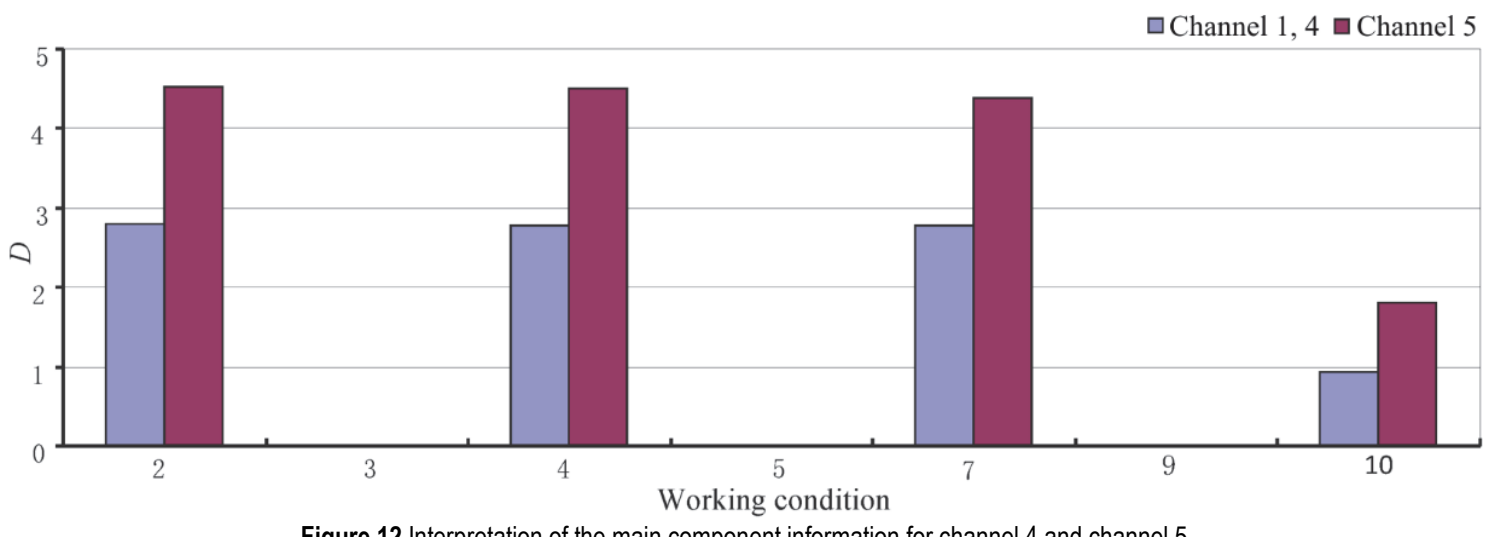

Figure 12 Interpretation of the main component information for channel 4 and channel 5

From the analysis results in Fig. 12, it can be obtained that the main component scores of the four channels of the working conditions 1 to 9 are all negative. In working condition 2 to condition 10, the main component scores of channel 2 and channel 3 are negative, and the main component scores of channel 4 and channel 5 are positive. Since working condition 1 to working condition 9 are structurally non-damaged, the calculated values correspond one-to-one with the experimental results. Working condition 2 to working condition 10 indicate that the structure is damaged, so it can be judged that the damage source is close to the $4^{\text {th }}$ and $5^{\text {th }}$ channels, and the positioning effect is significant.

It can be seen from the figure that the distance values of the four channels of the working condition 1 to working conditions 9 are very different from the working condition 2 to working condition 10, and the positioning effect is good. However, because the Markov distance values of the 10 operating conditions are of approximate magnitude, the Markov distance values of the $4^{\text {th }}$ and $5^{\text {th }}$ channels in the 
working condition 10 are very close to the Markov distance values of the first nine working conditions, which can be approximated as a non-destructive state. There is a certain deviation from the experimental condition setting. By comparing the positioning results in Fig. 11 and Fig. 12, it can be seen that using the root-mean-square error (RMSE) of the AR model as the damage sensitivity index, the principal component analysis method can obtain higher damage identification accuracy.

\section{CONCLUSION}

The bridge health monitoring system is developing in the direction of networked data transmission and storage, integrated management of monitoring projects, intelligent monitoring of monitoring instruments, and real-time interaction. However, the research on modern concrete bridge health monitoring system is not deep enough, and there is still a long way to go in practical engineering applications. Due to the high cost of the bridge health monitoring system, only some large bridges supported by large projects are running the health monitoring system, and the versatility is not high. This paper reveals the monitoring data processing from the perspective of signal processing and data mining. Faced with a large amount of bridge monitoring data, there is no relatively perfect processing method, which makes a large amount of data accumulated and piled up.

The original bridge morphology monitoring data obtained by the modern concrete bridge structural health monitoring is based on damage recognition. The noise reduction and motion information extraction are used to analyze the sequence data, which constructs a deformed sample data set and uses hierarchical thinking to sequentially establish data sampling. The modern concrete bridge structure health monitoring system based on damage recognition proposed in this paper is learned and identified by the intelligent sensing network to obtain the full bridge geometry data that fits the actual conditions of the test bridge. In agreement, the trend curve of the test bridge under each working condition is the same, and the accuracy meets the needs of engineering practice.

\section{Acknowledgement}

This paper is supported by the National Natural Science Foundation of China (51808259).

\section{REFERENCES}

[1] An, Y., Chatzi, E., Sim, S. H., Laflamme, S., Blachowski, B., \& Ou, J. (2019). Recent progress and future trends on damage identification methods for bridge structures. Structural Control and Health Monitoring, 26(10), e2416. https://doi.org/10.1002/stc.2416

[2] Prendergast, L. J., Limongelli, M. P., Ademovic, N., Anžlin, A., Gavin, K., \& Zanini, M. (2018). Structural health monitoring for performance assessment of bridges under flooding and seismic actions. Structural Engineering International, 28(3), 296-307. https://doi.org/10.1080/10168664.2018.1472534

[3] Ebrahimian, H., Astroza, R., Conte, J. P., \& Papadimitriou, C. (2018). Bayesian optimal estimation for output-only nonlinear system and damage identification of civil structures. Structural Control \& Health Monitoring, 25(4). https://doi.org/10.1002/stc.2128

[4] Panesso, A. M., Marulanda, J., \& Thomson, P. (2019). Experimental evaluation of vibration based damage identification techniques on a pedestrian bridge. Dyna, 86(209), 9-16. https://doi.org/10.15446/dyna.v86n209.73351

[5] Xiaodan, F. (2017). Design and implementation of remote health monitoring system for $3 \mathrm{D}$ visual bridge. Procedia engineering, 174, 1330-1335. https://doi.org/10.1016/j.proeng.2017.01.282

[6] Li, H. N., Ren, L., Jia, Z. G., Yi, T. H., \& Li, D. S. (2016). State-of-the-art in structural health monitoring of large and complex civil infrastructures. Journal of Civil Structural Health Monitoring, 6(1), 3-16. https://doi.org/10.1007/s13349-015-0108-9

[7] Abdeljaber, O., Avci, O., Kiranyaz, S., Gabbouj, M., \& Inman, D. J. (2017). Real-time vibration-based structural damage detection using one-dimensional convolutional neural networks. Journal of Sound and Vibration, 388, 154170. https://doi.org/10.1016/j.jsv.2016.10.043

[8] Cerda, F., Chen, S., Bielak, J., Garrett, J. H., \& Kovacevic, J. (2014). Indirect structural health monitoring of a simplified laboratory-scale bridge model. Smart Structures \& Systems, 13(5), 849-868. https://doi.org/10.12989/sss.2014.13.5.849

[9] He, L., Reynders, E. , Jaime H. García-Palacios, Marano, G. C., \& Roeck, G. D. (2020). Wireless-based identification and model updating of a skewed highway bridge for structural health monitoring. Applied Emcees, 10(7), 2347. https://doi.org/10.3390/app10072347

[10] Bas, S., Apaydin, N. M., Ilki, A., \& Catbas, F. N. (2018). Structural health monitoring system of the long-span bridges in Turkey. Structure and Infrastructure Engineering, 14(4), 425-444. https://doi.org/10.1080/15732479.2017.1360365

[11] Chen, Y.-X., Lu, P.-M., Guo, C.-J., Li, D.-T., \& Wang, B.H. (2014). Study on fatigue performance of orthotropic steel bridge deck u-rib and cover weld structure. Journal of Changan University, 34(1), 49-55.

[12] Zhang, L., Zhou, G., Han, Y., Lin, H., \& Wu, Y. (2018). Application of Internet of Things technology and convolutional neural network model in bridge crack detection. IEEE Access, 6, 39442-39451. https://doi.org/10.1109/ACCESS.2018.2855144

[13] Mukhopadhyay, T. (2018). A multivariate adaptive regression splines based damage identification methodology for web core composite bridges including the effect of noise. Journal of Sandwich Structures \& Materials, 20(7), 885903. https://doi.org/10.1177/1099636216682533

[14] Bao, Y., Shi, Z., Beck, J. L., Li, H., \& Hou, T. Y. (2017). Identification of time-varying cable tension forces based on adaptive sparse time-frequency analysis of cable vibrations. Structural Control and Health Monitoring, 24(3). https://doi.org/10.1002/stc.1889

[15] Li, L. \& Qin, H. (2018). An ukf-based nonlinear system identification method using interpolation models and backward integration. Structural Control \& Health Monitoring, 25(4), e2129.1-e2129.18. https://doi.org/10.1002/stc.2129

[16] Zhang, J. Yu, C.-C., \& Chen, P. (2010). Design and implementation of bridge structure health monitoring data analysis platform. Computer Engineering \& Design, 31(13), $1-5$.

[17] Ay, A. M., Khoo, S., \& Wang, Y. (2019). Probability distribution of decay rate: a statistical time-domain damping parameter for structural damage identification. Structural Health Monitoring, 18(1), 66-86. https://doi.org/10.1177/1475921718817336

[18] Du, D. C., Vinh, H. H., Trung, V. D., Hong Quyen, N. T., \& Trung, N. T. (2018). Efficiency of Jaya algorithm for solving 
the optimization-based structural damage identification problem based on a hybrid objective function. Engineering Optimization, 50(8), 1233-1251.

https://doi.org/10.1080/0305215X.2017.1367392

\section{Contact information:}

\section{Chunguang HANG}

School of Civil Engineering, Jiaying University,

Meizhou, China

\section{Xu LUO}

(Corresponding author)

School of Mechanics and Construction Engineering,

Jinan University,

Guangzhou, China

E-mail: tluoxu_jnu@163.com

\section{Xinping LI}

School of Civil Engineering and Transportation,

South China University of Technology,

Guangzhou, China

\section{Xinsha FU}

School of Civil Engineering and Transportation,

South China University of Technology,

Guangzhou, China

\section{Lixiong GU}

School of Civil Engineering and Transportation,

South China University of Technology,

Guangzhou, China

\section{Zhiyong OUYANG}

School of Civil Engineering, Jiaying University,

Meizhou, China 